\title{
Coordinated Transport of a Slung Load by a Team of Autonomous Rotorcraft
}

\author{
ZuQun $\mathrm{Li}^{*}$ \\ Joseph F. Horn ${ }^{\dagger}$ \\ Jack W. Langelaan ${ }^{\ddagger}$ \\ The Pennsylvania State University, University Park, PA 16802, USA
}

\begin{abstract}
This paper describes a hierarchical approach to coordinated transportation of a slung load by a team of autonomous helicopters. A closed-loop controller computes net force and moments on the load's center of gravity so a desired trajectory is followed; cable forces at each attachment point are computed so that the net force and moment on the center of gravity equal the desired values from the controller; finally each helicopter's position, velocity, and thrust are computed to obtain the desired attachment force, assuming a compliant cable connects the helicopter to the load. Results of simulations showing four degree of freedom transport of a load (North, East, Down position and yaw angle) show the utility of the proposed approach.
\end{abstract}

\section{Introduction}

Rotorcraft are often the most effective way to transport cargo and crews to and from areas that fixed-wing aircraft cannot reach safely. However, the maximum load capacity of current rotorcraft is somewhat limited. Many heavy military vehicles and cargos cannot be transport by a single rotorcraft. While larger and higher lifting capacity rotorcraft could be developed to solve this problem, it is very inefficient to develop and manufacture huge rotorcraft whose full lift capacity will be rarely used. With the cooperation of multiple rotorcraft, transporting heavy cargos and vehicles can be much more economically and effectively achieved. This is known as multi-lift.

The concept of using two helicopters to cooperatively carry a single slung load (twin-lift) has been examined at several times over the past few decades for both manned and unmanned helicopters. ${ }^{1-4}$ However, pilot workload and vehicle safety considerations have precluded implementation except for some isolated demonstrations (Figure 1(a)). Operational development is hindered by overall system complexity and coordinating control along typical flight paths.

Most of the research conducted to date has focused on twin-lift, with equations of motion and equilibrium conditions discussed by Cicolani and Kanning; ${ }^{6,7}$ operational concepts such as spreader bars are also discussed (see Figure 1(b)). Research into control of twin-lift systems has examined synthesis, ${ }^{8}$ stability augmentation using non-linear state feedback ${ }^{9,10}$ and adaptive control. ${ }^{11}$

This paper is focused on the more general problem of multi-lift, using two or more autonomous rotorcraft to cooperatively carry a single payload. It seeks to develop a solution that is scalable at least up to reasonable numbers of rotorcraft (and here uses a group of four helicopters as the motivating scenario). The solution is a hierarchical approach, based on a concept known as Object Based Task Level Control (OBTLC), developed for robotic manipulator systems. ${ }^{12}$ In this framework top-level control is abstracted to the level of the desired payload trajectory and lower-level controllers onboard each robot determine the individual control inputs required to ensure that the desired payload trajectory is followed. In the application examined here, top level control is a trajectory following controller that computes desired payload acceleration, a middle layer computes the cable force that will result in the desired acceleration, and a low-level controller aboard

*Graduate Student, Department of Aerospace Engineering, Student Member AIAA.

${ }^{\dagger}$ Associate Professor, Department of Aerospace Engineering, Associate Fellow AIAA.

${ }^{\ddagger}$ Associate Professor, Department of Aerospace Engineering, Associate Fellow AIAA. 


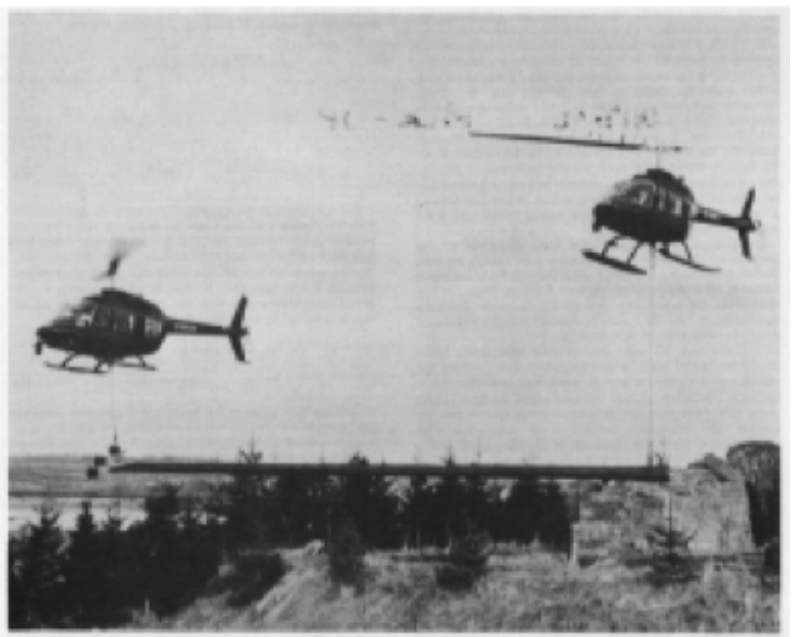

(a)

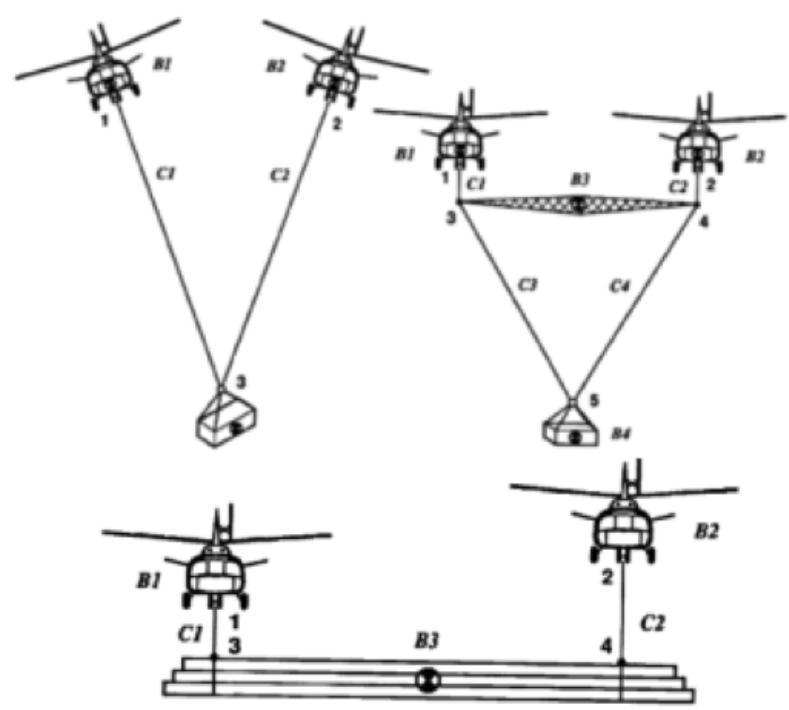

(b)

Figure 1. Left: flight demonstration using manned rotorcraft; Right: operational concepts. ${ }^{5}$

each helicopter ensures that the required cable tension and cable angle is actually flown. This approach has several advantages: it is scalable, allowing the team (or flock) of rotorcraft to grow as the payload weight increases; it is possible to bring a human into the loop at several levels (for example, a human operator could "steer the payload" while the rotorcraft steer themselves in a way that best allows the payload to follow commanded inputs; a human could also take over at the level of rotorcraft control, although this could be difficult for a human pilot); finally, the abstractions at each level mean that the implementation details at each level do not have a significant effect (beyond performance constraints) on other parts of the control architecture.

The remainder of this paper is organized as follows. Section II provides an overview of the problem at hand, defining coordinates, describing the control methodology and defining dynamics of the payload. Section III discusses each of the components of the control system, focusing especially on computing the cable forces that are required for the payload to follow a desired trajectory while fulfilling constraints such as helicopter separation. Section IV discusses the simulation used to show the utility of the approach, and Section V presents results of the simulation. Finally, some concluding remarks are presented in Section VI.

\section{Problem Description}

The goal here is to control a load carried by $N$ helicopters so that the payload will follow its desired trajectory (Figure 2). It is assumed that each helicopter has knowledge of its own state and that payload state is also known (e.g. via GPS/INS).

Referring to Figure 2, payload position $\mathbf{p}$ is expressed in an inertial North-East-Down frame $O$, and the desired trajectory is defined in this frame. Cable attachment points $\mathbf{g}_{i}$ are defined in the payload-fixed frame, and the position $\mathbf{r}_{i}$ of a helicopter is expressed in the inertial frame.

Given a desired trajectory, the required forces $\mathbf{F}_{C G}$ and moments $\mathbf{M}_{C G}$ to ensure that the trajectory is flown can be computed from payload dynamics. The problem is now to determine the cable forces $\mathbf{F}_{i}$ so that: (1) the sum of forces and moments induced by cable forces at the payload CG is equal to the desired forces and moments; (2) vehicle separation constraints are satisfied; (3) other constraints (such as controllability or disturbance rejection) are satisfied.

While trajectory generation is not the subject of this paper, It is assumed that the desired trajectory is dynamically and kinematically feasible: i.e. each helicopter is able to generate the required thrust to maintain desired cable force, and each helicopter is equipped with a flight controller so that desired helicopter state is maintained. Issues related to helicopter control are addressed in other work. ${ }^{13}$ 


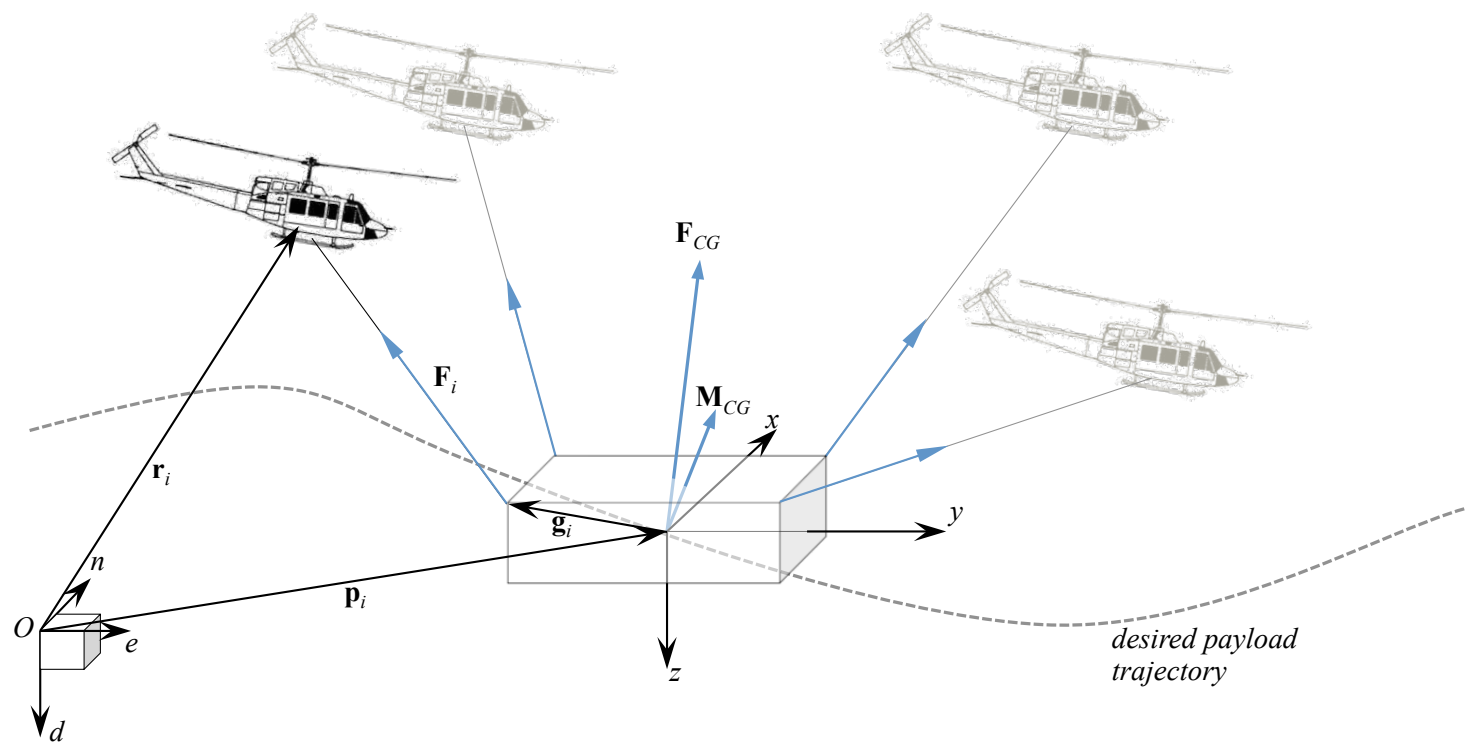

Figure 2. Schematic of coordinated slung-load transport problem.

\section{A. System Description and Control Architecture}

The block diagram in Figure 3 shows a hierarchical approach to coordinated transport.

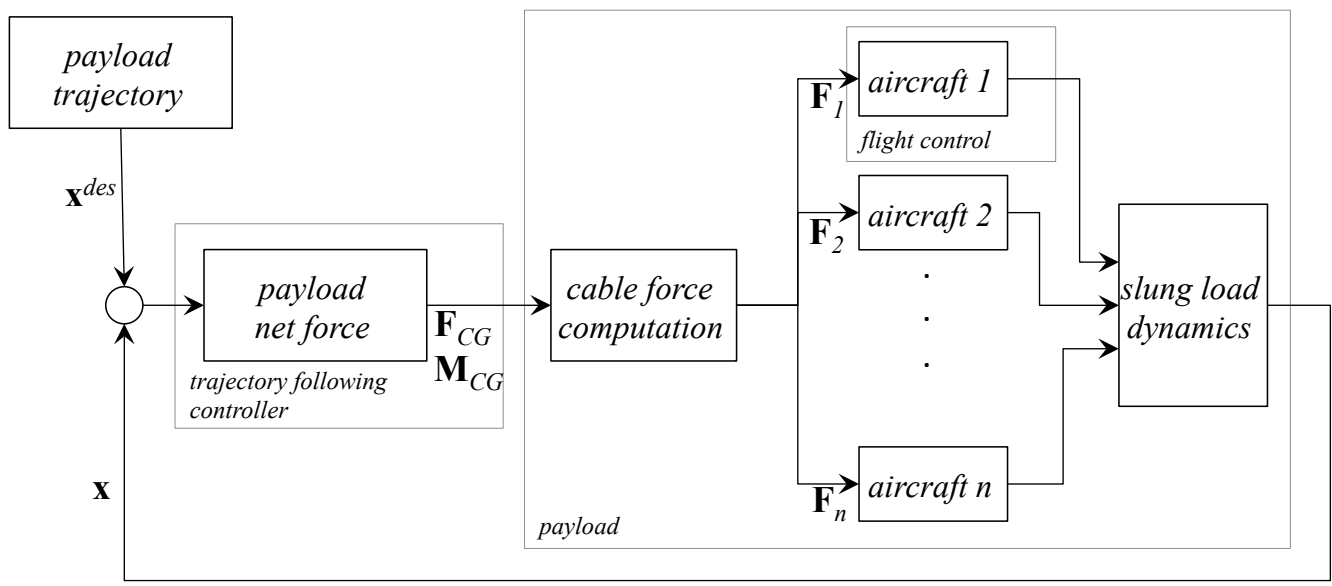

Figure 3. Schematic of Object Based Task Level Control for coordinated transport of slung load.

The desired payload state $\mathbf{x}^{\text {des }}$ is obtained from some payload trajectory (or human operator). A trajectory following controller computes the desired net force $\mathbf{F}_{C G}$ and moment $\mathbf{M}_{C G}$ acting on the payload (equivalently, the net acceleration and net angular acceleration acting about the center of gravity of the payload). Cable forces $\mathbf{F}_{i}$ that result in this net force and moment are computed based on the geometry of the cable attachments and constraints such as vehicle separation, and a flight controller aboard each helicopter ensures that the required cable tension and cable angles (with respect to the payload) are flown. Payload state $\mathbf{x}$ is provided either by a sensor (e.g. GPS/INS) on the payload or it is estimated by the team of helicopters. 


\section{B. Payload Dynamics}

Payload position is expressed in the North-East-Down frame as $n, e, d$. Payload rotations are expressed as Euler angles $\phi, \theta, \psi$ relative to the fixed North-East-Down frame. Payload velocities $u, v, w$ are expressed in the payload body frame. Using the standard definition of Euler angles, payload kinematics are

$$
\begin{aligned}
\dot{n} & =u \cos \theta \cos \psi+v(\sin \phi \sin \theta \cos \psi-\cos \phi \sin \psi)+w(\cos \phi \sin \theta \cos \psi+\sin \phi \sin \psi) \\
\dot{e} & =u \cos \theta \sin \psi+v(\sin \phi \sin \theta \sin \psi+\cos \phi \cos \psi)+w(\cos \phi \sin \theta \sin \psi-\sin \phi \cos \psi) \\
\dot{d} & =-u \sin \theta+v \sin \phi \cos \theta+w \cos \phi \cos \theta \\
\dot{\phi} & =p+q \sin \phi \tan \theta+r \cos \psi \tan \theta \\
\dot{\theta} & =q \cos \phi-r \sin \phi \\
\dot{\psi} & =q \frac{\sin \phi}{\cos \theta}+r \frac{\cos \phi}{\cos \theta}
\end{aligned}
$$

Assuming that the payload's mass moment of inertia matrix is diagonal,

$$
\begin{aligned}
\dot{u} & =r v-q w-g \sin \theta+\frac{F_{x}}{m} \\
\dot{v} & =p w-r u+g \cos \theta \sin \phi+\frac{F_{y}}{m} \\
\dot{w} & =q u-p v+g \cos \theta \cos \phi+\frac{F_{z}}{m} \\
\dot{p} & =\frac{1}{J_{x} J_{z}}\left[J_{z} M_{x}-\left(J_{z}^{2}-J_{z} J_{y}\right) q r\right] \\
\dot{q} & =\frac{1}{J_{y}}\left[\left(M_{y}+\left(J_{z}-J_{x}\right) r p\right]\right. \\
\dot{r} & =\frac{1}{J_{x} J_{z}}\left[J_{x} M_{z}+\left(J_{x}^{2}-J_{x} J_{y}\right) p q\right]
\end{aligned}
$$

where $\mathbf{F}=\left[\begin{array}{lll}F_{x} & F_{y} & F_{z}\end{array}\right]^{T}$ (the net force acting on the payload CG, expressed in the body frame) and $\mathbf{M}=$ $\left[\begin{array}{lll}M_{x} & M_{y} & M_{z}\end{array}\right]^{T}$ (the net moment acting about the payload CG, expressed in the body frame).

\section{Cable Force}

A cable attached to the payload at a point $\mathbf{g}_{i}$ (see Figure 2) induces a force and moment on the CG:

$$
\left[\begin{array}{c}
\mathbf{F}_{C G, i} \\
\mathbf{M}_{C G, i}
\end{array}\right]=\left[\begin{array}{c}
\mathbf{F}_{i} \\
\mathbf{g}_{i} \times \mathbf{F}_{i}
\end{array}\right]
$$

Written as a matrix multiplication,

$$
\left[\begin{array}{c}
\mathbf{F}_{C G, i} \\
\mathbf{M}_{C G, i}
\end{array}\right]=\left[\begin{array}{ccc}
1 & 0 & 0 \\
0 & 1 & 0 \\
0 & 0 & 1 \\
0 & -g_{i, z} & g_{i, y} \\
g_{i, z} & 0 & -g_{i, x} \\
-g_{1, y} & g_{1, x} & 0
\end{array}\right] \mathbf{F}_{i}=\mathbf{G}_{i} \mathbf{F}_{i}
$$

where $\mathbf{g}_{i}=\left[\begin{array}{lll}g_{i, x} & g_{i, y} & g_{i, z}\end{array}\right]^{T}$ defines the vector from the payload CG to the $i^{\text {th }}$ cable attachment point, $\mathbf{G}_{i}$ is a geometry matrix for the $i^{\text {th }}$ cable attachment (that defines the effect of the $i^{\text {th }}$ cable force on the CG), and $\mathbf{F}_{i}=\left[\begin{array}{lll}F_{i, x} & F_{i, y} & F_{i, z}\end{array}\right]^{T}$ are the components of the cable tension. Note that the choice of frame in which $\mathbf{g}_{i}$ and $\mathbf{F}_{i}$ are resolved is arbitrary (although both must be resolved in the same frame), it is in practice most convenient to resolve these in the payload body frame. 
The total force and moment acting at the payload CG is the sum of contributions from all the cables:

$$
\begin{aligned}
{\left[\begin{array}{c}
\mathbf{F}_{C G} \\
\mathbf{M}_{C G}
\end{array}\right] } & =\left[\begin{array}{llll}
\mathbf{G}_{1} & \mathbf{G}_{2} & \cdots & \mathbf{G}_{N}
\end{array}\right]\left[\begin{array}{c}
\mathbf{F}_{1} \\
\mathbf{F}_{2} \\
\vdots \\
\mathbf{F}_{N}
\end{array}\right] \\
& =\mathbf{G F}_{\text {cable }}
\end{aligned}
$$

A six degree of freedom payload will require at least three cables to provide control over all degrees of freedom. While two cables will give six components of cable force (and thus a square $\mathbf{G}$ matrix), a solution for $\mathbf{F}_{\text {cable }}$ will not exist because $\mathbf{G}$ will be singular. Physically, this condition will leave the payload free to rotate about the vector connecting the two attachment points. With three or more cables Equation 16 is underdetermined, giving an infinite number of solutions. This property will be used to compute cable forces that satisfy the desired net force and moment while simultaneously satisfying other constraints. A method for solving Equation 16 is discussed in a later section.

\section{Helicopter Desired State and Acceleration}

The components of cable force computed from the solution to Equation 16 define cable angles with respect to the payload body frame. Combined with cable length, these angles define the desired position of a helicopter with respect to the payload; helicopter velocity and acceleration with respect to the payload are defined by desired position and the payload trajectory. The helicopter's net thrust vector is determined by the desired cable force and by desired helicopter acceleration. This is shown schematically in Figure 4.

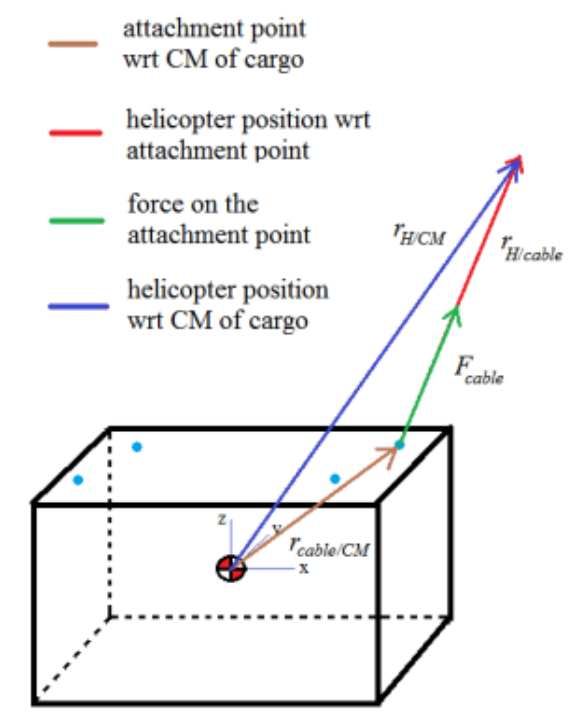

Figure 4. Schematic of Helicopter position with respect to CG of payload.

A cable is modeled as a damped spring, so the magnitude of tension in the $i^{\text {th }}$ cable is

$$
F_{i}=k_{c} \Delta l_{i}+c_{c} \dot{l}_{i}
$$

where $\Delta l_{i}$ is the difference between stretched length and unstretched length of the cable, $k_{c}$ is the spring constant of the cable, $i_{i}$ is the rate of change of cable length, and $c_{c}$ is the damping constant of the cable. Clearly cable tension must be non-negative (you can't push on a rope).

Given net cable length, the position of the $i^{\text {th }}$ helicopter relative to the attachment point is

$$
\mathbf{r}_{\text {heli } / a t t, i}=\left(l_{i, 0}+\Delta l_{i}\right) \frac{\mathbf{F}_{i}}{F_{i}}
$$


where $l_{i, 0}$ is the unstretched cable length. The position of a helicopter with respect to the payload is

$$
\mathbf{r}_{i}^{C G}=\mathbf{g}_{i}+\mathbf{r}_{i}^{a t t}
$$

Then the desired position, velocity, and acceleration of the $i^{\text {th }}$ helicopter relative to the inertial frame are

$$
\begin{aligned}
\mathbf{r}_{i} & =\mathbf{p}+\mathbf{g}_{i}+\mathbf{r}_{i}^{C G} \\
\mathbf{v}_{i} & =\mathbf{v}+i_{i} \frac{\mathbf{F}_{i}}{\left|F_{i}\right|}+\boldsymbol{\omega} \times \mathbf{r}_{i}^{C G} \\
\mathbf{a}_{i} & =\mathbf{a}+\dot{\boldsymbol{\omega}} \times \mathbf{r}_{i}^{C G}+\boldsymbol{\omega} \times \boldsymbol{\omega} \times \mathbf{r}_{i}^{C G}
\end{aligned}
$$

\section{Coordinated Transport}

To illustrate the control architecture proposed here, a payload trajectory controller consisting of feedforward accelerations and state feedback is used to compute desired payload forces and moments. A general method for computing cable forces is derived: this method first computes a least norm solution for the required cable forces, and then uses the null space of the cable geometry matrix to ensure that constraints are satisfied. This guarantees that cable forces satisfy the desired net payload forces.

\section{A. Payload Trajectory Control}

In the transport strategy proposed here the first step is controlling payload state. This may involve maintaining position over a target or the more general case following a desired trajectory, and the specific choice of trajectory following controller is arbitrary. Recall that the output of the trajectory following controller is a desired net force and moment on the payload center of gravity (or equivalently, desired payload acceleration). For demonstration purposes a controller that follows a trajectory in an inertial reference frame is used here.

The payload is assumed to be near level (i.e. pitch and roll angles are small) and angular rates are small. Payload kinematics are therefore

$$
\begin{aligned}
\dot{n} & =v_{n} \\
\dot{e} & =v_{e} \\
\dot{d} & =v_{d} \\
\dot{\phi} & =p \\
\dot{\theta} & =q \\
\dot{\psi} & =r \\
\dot{v}_{n} & =\frac{F_{n}}{m} \\
\dot{v}_{e} & =\frac{F_{e}}{m} \\
\dot{v}_{d} & =g+\frac{F_{d}}{m} \\
\dot{p} & =\frac{M_{x}}{J_{x}} \\
\dot{q} & =\frac{M_{y}}{J_{y}} \\
\dot{r} & =\frac{M_{z}}{J_{z}}
\end{aligned}
$$

where $v_{(\cdot)}$ denotes components of velocity in the north, east, or down direction and $F_{(\cdot)}$ denotes components of the net force in the north, east or down directions. Written compactly in discrete form,

$$
\mathbf{x}_{k+1}=\mathbf{A} \mathbf{x}_{k}+\mathbf{B u}
$$


where

$$
\begin{aligned}
& \mathbf{x}_{k}=\left[\begin{array}{lllllllllll}
n_{k} & e_{k} & d_{k} & \phi_{k} & \theta_{k} & \psi_{k} & v_{n, k} & v_{e, k} & v_{d, k} & p_{k} & q_{k} \\
r_{k}
\end{array}\right]^{T} \\
& \mathbf{u}_{k}=\left[\begin{array}{llllll}
F_{n, k} & F_{e, k} & F_{d, k} & M_{x, k} & M_{y, k} & M_{z, k}
\end{array}\right]^{T}
\end{aligned}
$$

For trajectory following a controller of the form

$$
\mathbf{u}_{k}=\mathbf{K}\left(\mathbf{x}_{k, \text { des }}-\mathbf{x}_{k}\right)+\mathbf{u}_{k, t r a j}
$$

is used, where $\mathbf{x}_{k, \text { des }}$ is the desired payload state at time step $k$ and $\mathbf{u}_{k, t r a j}$ is a feed forward term computed from the desired acceleration $\mathbf{a}_{t r a j}$ of payload on the trajectory and the payload inertia matrix $\mathbf{M}$. The feedback gain $\mathbf{K}$ can be computed using any approach; for the simulation results presented later LQR synthesis is used for convenience. A schematic of this controller is shown in Figure 5.

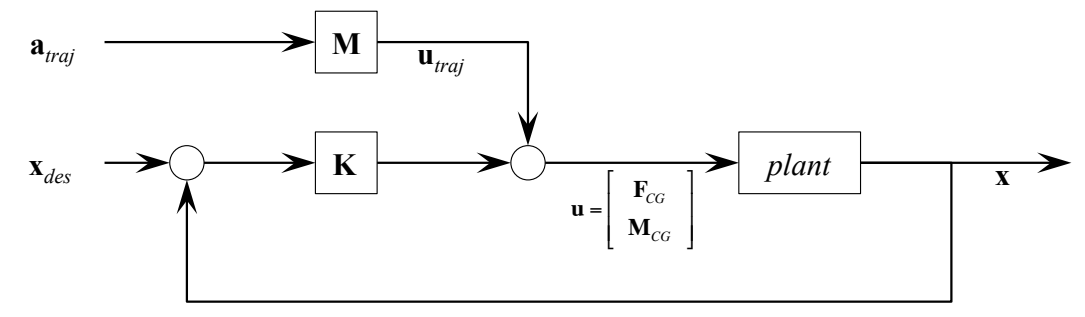

Figure 5. Block diagram of feedforward and state feedback controller used here.

The desired payload forces computed above are expressed in the inertial frame (moments are in the payload body frame). In the body frame, payload forces and moments are

$$
\left[\begin{array}{c}
\mathbf{F}_{C G} \\
\mathbf{M}_{C G}
\end{array}\right]=\left[\begin{array}{cc}
\mathbf{T} & \mathbf{0} \\
\mathbf{0} & \mathbf{I}
\end{array}\right] \mathbf{u}
$$

where $\mathbf{T}$ is the direction cosine matrix that transforms a vector from the inertial frame to the body frame and $\mathbf{0}$ and $\mathbf{I}$ are a $3 \times 3$ matrix of zeros and the identity matrix, respectively.

The desired payload forces and moments can now be used to compute the desired cable forces.

\section{B. A general formulation for cable force computation}

As stated earlier, Equation 16 is underdetermined, hence there are an infinite number of solutions. One solution minimizes the total cable forces (the minimum norm solution), and this can be computed in closed form given a particular cable attachment geometry matrix $\mathbf{G}$ :

$$
\mathbf{F}_{\text {cable }}^{L N}=\mathbf{G}^{T}\left(\mathbf{G G}^{T}\right)^{-1}\left[\begin{array}{c}
\mathbf{F}_{C G} \\
\mathbf{M}_{C G}
\end{array}\right]
$$

In the payload body frame $\mathbf{G}$ is constant, thus $\mathbf{G}^{T}\left(\mathbf{G G}^{T}\right)^{-1}$ can be precomputed. Computing a set of cable forces that satisfy the desired net forces can thus happen at high rate in real time.

This least norm solution will ensure that the desired net force and moment acting on the payload center of gravity is satisfied. However, constraints (such as helicopter separation) or other considerations (such as controllability of the payload) may also apply. Cable forces that exist in the null space of $\mathbf{G}$ will not affect payload net forces and moments, and the null space can be used to satisfy constraints or other considerations. The net cable force vector will thus be

$$
\mathbf{F}_{\text {cable }}=\mathbf{F}_{\text {cable }}^{L N}+\mathbf{F}_{\text {cable }}^{\text {null }}
$$

where (by definition)

$$
\mathbf{G F}_{\text {cable }}^{\text {null }}=0
$$


A set of forces that satisfy Equation 42 can be computed as a linear combination of vectors that span the nullspace of $\mathbf{G}$. Suppose $\left\{\tilde{\mathbf{g}}_{i}, i=1 \ldots 3 N-6\right\}$ define an orthonormal basis for the nullspace of $\mathbf{G}$, then

$$
\mathbf{F}_{\text {cable }}^{\text {null }}=\left[\begin{array}{llll}
\tilde{\mathbf{g}}_{1} & \tilde{\mathbf{g}}_{2} & \cdots & \tilde{\mathbf{g}}_{3 N-6}
\end{array}\right] \mathbf{f}
$$

will automatically exist in the nullspace of $\mathbf{G}$ and will thus have zero net effect on the payload center of gravity. The problem now is to find $\mathbf{f}$ so that constraints are satisfied.

This can be done by solving the optimization problem

$$
\begin{aligned}
\operatorname{minimize} & C\left(\mathbf{F}_{\text {cable }}^{L N}, \mathbf{f}\right) \\
\text { subject to } & \mathbf{F}_{\text {cable }}=\mathbf{F}_{\text {cable }}^{L N}+\tilde{\mathbf{G}} \mathbf{f} \\
& g\left(\mathbf{F}_{\text {cable }}\right) \leq 0 \\
& h\left(\mathbf{F}_{\text {cable }}\right)=0 \\
& 0<F_{i} \leq F_{\max }
\end{aligned}
$$

The procedure for computing the required cable forces is thus to first compute the least norm solution and then compute the null space forces so that constraints are satisfied. The cost function (Equation 44) defines the additional considerations (e.g. controllability) or tries to minimize total cable force; the inequality and equality constraints (Equations 46 and 47) can be used to impose separation constraints by ensuring that cable force vectors are pointing in appropriate directions; the magnitude constraint (Equation 48) ensures that positive tension is maintained on each cable and that maximum allowable tension is not exceeded.

\section{Helicopter control}

A critical component is the helicopter's on-board control. It has two main functions: first, to ensure that the desired vehicle state is maintained; second, to ensure that the desired cable tension is maintained. Helicopter control is not the focus of this paper, but a few criteria are briefly outlined.

The required accuracy of helicopter state control is dependent on cable length. Since the position of the helicopter with respect to the payload defines the direction of the cable force vector, a long cable will result in less sensitivity to errors in helicopter relative position. Longer cables will also result in a longer system time constant. Cable extensibility will also permit more error in helicopter state control, however the question of stability as a function of cable stiffness must be addressed.

Cable tension is critical. Loss of tension in a cable may result in loss of the payload (and of the vehicles transporting the payload). A means to measure cable tension as well as fast response to commanded changes in helicopter thrust will greatly improve overall performance.

\section{Payload state computation}

Here it is assumed that payload state is known through a GPS/INS mounted on the payload. If this system is not available then a means of estimating payload state from helicopter state will be required.

\section{Multi-Lift Simulation}

While the control methodology described above is scalable to (in principle) any size of flock, here a group of four helicopters cooperatively transporting a payload is discussed as the motivating example.

Figure 6 shows a block diagram of the simulation. Each helicopter is connected to the payload by a cable (modeled as a damped spring).

\section{A. Helicopter Simulation Model}

The focus of this paper is on payload control, thus a point mass model of the aircraft translational kinematics is coupled with a linear representation of the attitude and thrust dynamics. It is assumed that a dynamic inversion control law regulates the attitude and thrust dynamics to follow a linear command filter. An outer loop dynamic inversion control law was designed to track the desired position, velocity, and acceleration commands of each helicopter. Figure 7 shows the free body diagram of the helicopter. 


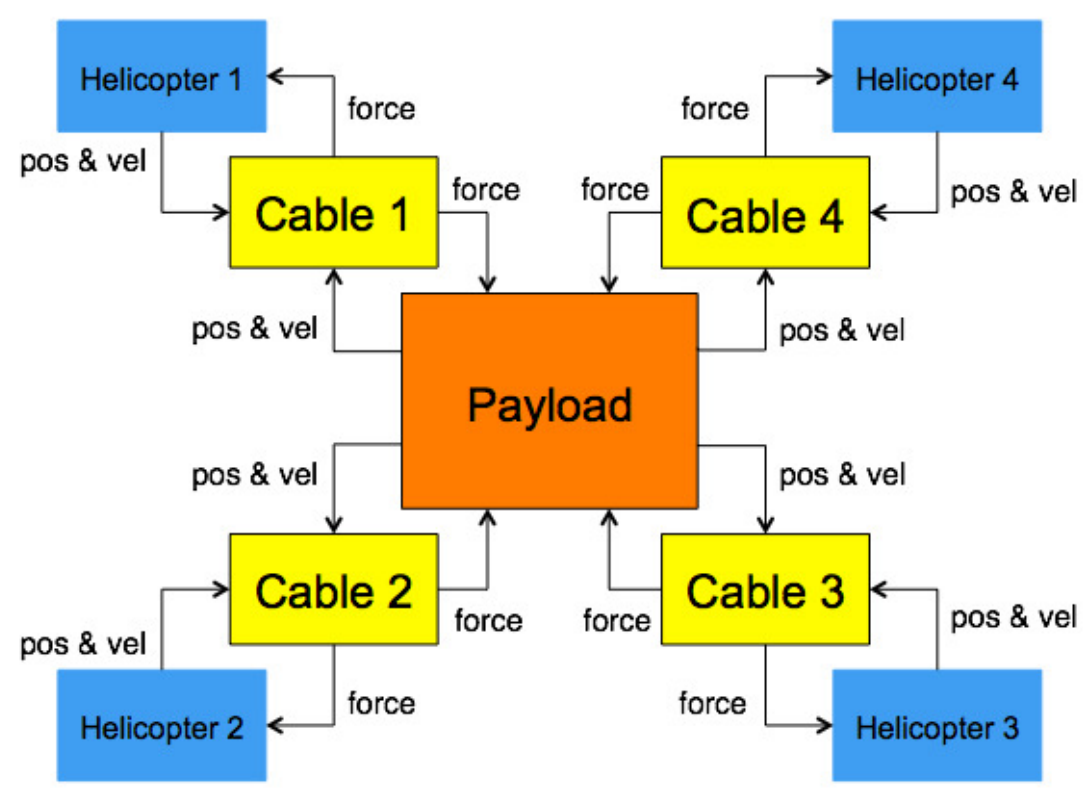

Figure 6. Multi-Lift Simulation Overview

Helicopter dynamics are second order in the closed loop:

$$
\left[\begin{array}{c}
\ddot{n}_{i} \\
\ddot{e}_{i} \\
\ddot{d}_{i}
\end{array}\right]=\frac{1}{m}\left(f\left(\phi_{c}, \theta_{c}, \psi_{c}, T_{c}\right)-\frac{1}{2} \rho f_{e} v_{a}\left[\begin{array}{c}
\dot{n}_{i} \\
\dot{e}_{i} \\
\dot{d}_{i}
\end{array}\right]-\mathbf{F}_{\text {cable }}\right)
$$

Inputs are Euler angles and total thrust, with Euler angles are filtered through a second order command filter and thrust filtered through a first order command filter.

$$
\begin{aligned}
\ddot{\phi}_{c}+2 \zeta_{\phi} \omega_{\phi} \dot{\phi}_{c}+\omega_{\phi}^{2}\left(\phi_{c}-\phi_{c m d}\right) & =0 \\
\ddot{\theta}_{c}+2 \zeta_{\theta} \omega_{\theta} \dot{\theta}_{c}+\omega_{\theta}^{2}\left(\theta_{c}-\theta_{c m d}\right) & =0 \\
\ddot{\psi}_{c}+2 \zeta_{\psi} \omega_{\psi} \dot{\psi}_{c}+\omega_{\psi}^{2}\left(\psi_{c}-\psi_{c m d}\right) & =0 \\
\tau_{T} \dot{T}_{c}+\left(T_{c}-T_{c m d}\right) & =0
\end{aligned}
$$

Filter parameters are dependent on the inner loop control law bandwidth, which in turn is limited by the dynamics of the specific vehicle. In the following simulations, bandwidth values were selected to match typical values for full scale rotorcraft.

\section{B. Cable Simulation Model}

The cable was modeled as spring damper system in the multi-lift simulation. However, unlike a spring damper system, the cable was modeled such that it has no resistance to compression. The magnitude of the cable force is

$$
F_{\text {cable }}=F_{\text {spring }}+F_{\text {damp }}
$$

where

$$
F_{\text {spring }}=\left\{\begin{array}{ll}
K_{c} \Delta l, & \text { if } \Delta l>0 \\
0, & \text { if } \Delta l \leq 0
\end{array} ; \quad F_{\text {damp }}= \begin{cases}C_{c} i, & \text { if } i>0 \\
0, & \text { if } i \leq 0 \text { or } \Delta l \leq 0\end{cases}\right.
$$

The direction of the cable force is computed from the position of the helicopter relative to the cable attachment point. 


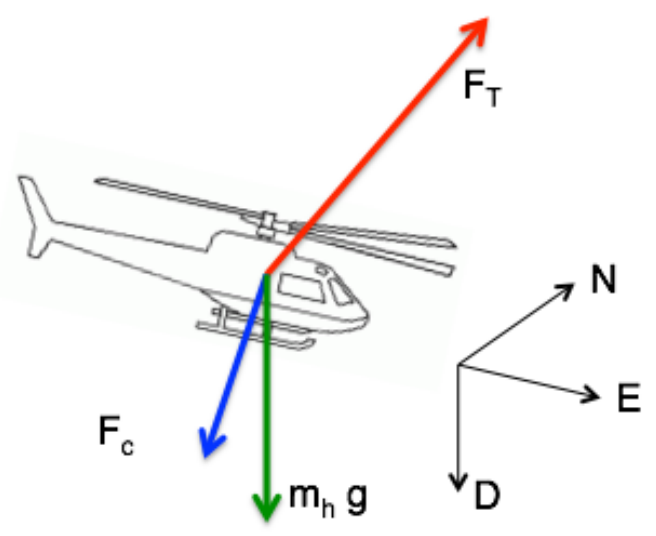

Figure 7. Free Body Diagram for Helicopter

\section{Payload Simulation Model}

The dynamics of payload was modeled as a rigid body with six degree of freedom using flat Earth assumption. The model uses the non-linear equations of motion that presented in Equation 1 to Equation 12, and these equation can be represented in compactly as:

$$
\dot{\mathbf{x}}=f\left(\mathbf{x}, \mathbf{F}_{C G}, \mathbf{M}_{C G}\right)+\frac{\mathbf{F}_{d r a g}}{m}+\frac{\mathbf{F}_{\text {wind }}}{m}
$$

where $\mathbf{F}_{\text {drag }}$ represents the drag force acting on the payload as it moves, and $\mathbf{F}_{\text {wind }}$ represents the force due to constant wind. The net payload forces are computed from the cable forces using Equation 16.

\section{Simulation Parameters}

Table 1 shows payload, cable, helicopter parameters, and environmental parameters used for the simulations. Here Kaman K-MAX is used as a representative autonomous helicopter.

In Table 1 payload mass, payload inertia, cable diameter, cable damping constant, and wind velocity will be different for different case of investigation.

The cable attachment points with respect to $\mathrm{CG}$ of payload in body frame for all cases are show in Table 2 .

\section{E. Constraints}

The cost function for cable force computation, defined in Equation 57, will try to minimize the total cable force, which will minimize fuel consumption for the helicopters.

$$
C\left(F_{\text {cable }}^{L N}, f\right)=\sqrt{\left|F_{\text {cable }, 1}\right|^{2}+\left|F_{\text {cable }, 2}\right|^{2}+\left|F_{\text {cable }, 3}\right|^{2}+\left|F_{\text {cable }, 4}\right|^{2}}
$$

where $F_{\text {cable }, i}$ is defined by Equation 41 .

Helicopter separation constraint was applied to ensure operational safety, and it is defined by inequality distance constraints shown in Figure 8. Where H1, H2, H3, and H4 defined in Fig. 8 are representing helicopters 1 to 4 .

Maximum cable force constraint was also applied to ensure the cable force for each attachment point is smaller than helicopter's external load capacity.

\section{Simulation Results}

Results of three cases are presented in the following subsections to demonstrate the performance of the proposed method and to compare stability between low helicopter separation and high separation. 
Table 1. Payload, Cable, and Helicopter parameters used for the investigated scenario.

\begin{tabular}{|c|c|c|c|}
\hline \multicolumn{4}{|c|}{ Payload Parameter } \\
\hline Payload Mass (kg) & vary & Width $(\mathrm{m})$ & 2.438 \\
\hline Length (m) & 6.058 & Height (m) & 2.591 \\
\hline Frontal Area $\left(m^{2}\right)$ & 6.319 & Drag Coeff & 1 \\
\hline$I_{\text {load }}\left(k g * m^{2}\right)$ & vary & & \\
\hline \multicolumn{4}{|c|}{ Cable Parameter } \\
\hline Neutral Length (m) & 30 & Young's Modulus $\left(\mathrm{N} / \mathrm{mm}^{2}\right)$ & 150000 \\
\hline Cable diameter (mm) & vary & Damping Constant (N-s/m) & vary \\
\hline \multicolumn{4}{|c|}{ K-MAX ${ }^{14}$} \\
\hline Helicopter Mass (kg) & 2334 & & \\
\hline Payload capacity (kg) & 2722 & Rotor diameter $(\mathrm{m})$ & 8.4 \\
\hline Length $(\mathrm{m})$ & 15.8 & Height (m) & 4.14 \\
\hline$\omega_{\phi}, \omega_{\theta}$ & $4 \mathrm{rad} / \mathrm{s}$ & $\omega_{\psi}$ & $1 \mathrm{rad} / \mathrm{s}$ \\
\hline$\zeta_{\phi}, \zeta_{\theta}, \zeta_{\psi}$ & 0.9 & $\tau_{T}$ & 0.25 \\
\hline \multicolumn{4}{|c|}{ Environment Parameter } \\
\hline Air density $\left(\mathrm{kg} / \mathrm{m}^{3}\right)$ & 1.225 & Gravity $\left(m / s^{2}\right)$ & 9.81 \\
\hline Wind Velocity $(\mathrm{m} / \mathrm{s})$ & & [vary] & \\
\hline
\end{tabular}

Table 2. Attachment Relative Position with Respect to CG of Payload

\begin{tabular}{|c|c|c|c|}
\hline Attachment 1 & $g_{x}=-2 \mathrm{~m}$ & $g_{y}=-2 \mathrm{~m}$ & $g_{z}=-2 \mathrm{~m}$ \\
Attachment 2 & $g_{x}=-2 \mathrm{~m}$ & $g_{y}=2 \mathrm{~m}$ & $g_{z}=-2 \mathrm{~m}$ \\
Attachment 3 & $g_{x}=2 \mathrm{~m}$ & $g_{y}=2 \mathrm{~m}$ & $g_{z}=-2 \mathrm{~m}$ \\
Attachment 4 & $g_{x}=2 \mathrm{~m}$ & $g_{y}=-2 \mathrm{~m}$ & $g_{z}=-2 \mathrm{~m}$ \\
\hline
\end{tabular}

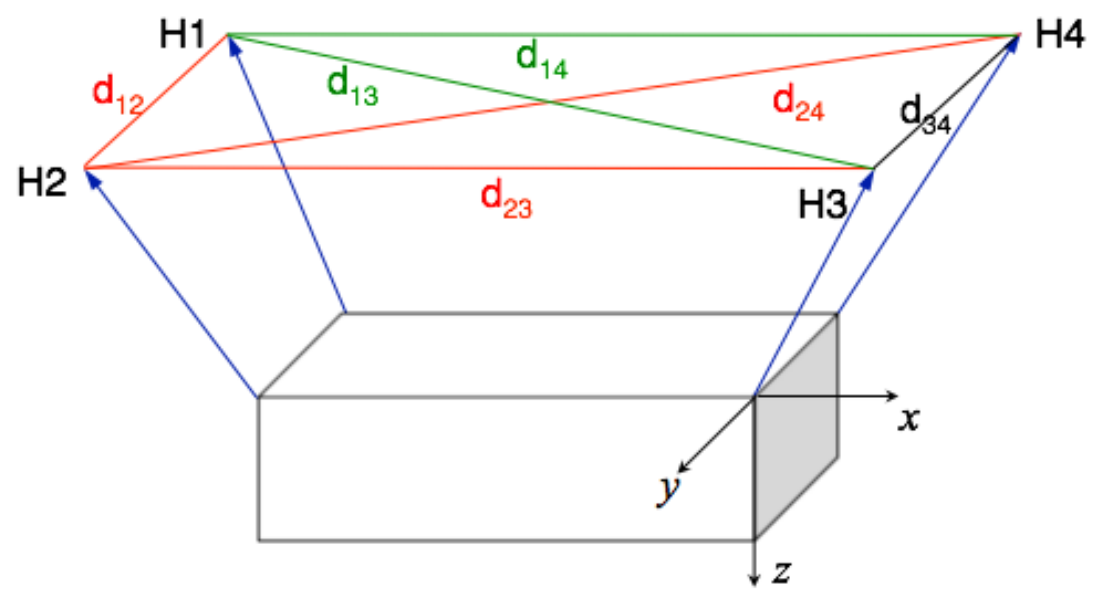

Figure 8. Distance constraint definition. $x-y-z$ is the payload body frame. 


\section{A. Combining linear and rotational payload motion}

The first case is a combination of translation and rotation maneuvers. A $5000 \mathrm{~kg}$ payload supported by $16 \mathrm{~mm}$ steel wire rope was used in this simulation. Table 3 shows the payload trajectory, helicopter separation constraint, and wind velocity for this case.

Table 3. Noise, trajectory, constraints, and wind velocity for the combined transportation case

\begin{tabular}{|c|c|c|c|}
\hline \multicolumn{4}{|c|}{ Initial and final states } \\
\hline Initial pos $(\mathrm{m})[\mathrm{t}=0]$ & 0 & 0 & 0 \\
\hline Initial vel $(\mathrm{m} / \mathrm{s})[\mathrm{t}=0]$ & 0 & 0 & 0 \\
\hline 1st point $(\mathrm{m})[\mathrm{t}=30]$ & 0 & 0 & 0 \\
\hline 2nd point $(\mathrm{m})[\mathrm{t}=90]$ & 0 & 0 & -500 \\
\hline 3rd point $(\mathrm{m})[\mathrm{t}=100]$ & 50 & 0 & -500 \\
\hline$* 4$ th point $(\mathrm{m})[\mathrm{t}=450]$ & 2912.5 & 0 & -500 \\
\hline Final pos $(\mathrm{m})[\mathrm{t}=540]$ & 2912.5 & 0 & -500 \\
\hline Final vel $(\mathrm{m} / \mathrm{s})[\mathrm{t}=540]$ & 0 & 0 & 0 \\
\hline Initial $\psi(\operatorname{deg})$ & 0 & 4th and final point $\psi(\mathrm{deg})$ & 45 \\
\hline Initial $\theta \& \psi(\operatorname{deg})$ & 0 & Final $\theta \& \psi(\operatorname{deg})$ & 0 \\
\hline Initial Angular rate $(\mathrm{deg} / \mathrm{s})$ & 0 & Final Angular rate (deg/s) & 0 \\
\hline Total sim time (sec) & 600 & & \\
\hline \multicolumn{4}{|c|}{ Helicopter Separation Constraint } \\
\hline$d_{12}, d_{23}, d_{34}, d_{14}(m)$ & 38 & $d_{13}, d_{24}(m)$ & 53.74 \\
\hline Wind Velocity $(\mathrm{m} / \mathrm{s})$ & -10 & 0 & 0 \\
\hline
\end{tabular}

Figure 9 shows the desired state history of the payload. The payload will first be lifted up to a height of 500 meters and then transported to a distance of roughly 2900 meters towards the north. When the payload arrives at its destination, it will hold its position while changing its heading angle from 0 degrees to 45 degrees. After the heading rotation is complete, the payload will hold its final state for 60 seconds to show the decay in state error.

Figure 10 shows the payload state error. The maximum translational error is about three meters, which occurs at beginning of the simulation (in the down coordinate) due to imperfect initialization. The maximum rotational error is in pitch, and has a magnitude of about 0.75 degrees. This occurs during the acceleration phase: the two leading helicopters have to pull harder to increase the North component cable force. However, this action also increases their upwards component of cable force, so the rear helicopters have to decrease their cable force in order to maintain the vertical position. This action induce a torque that pitches the payload. During the yaw rotation period, the error for yaw angular rate has high frequency and is oscillating. The cause of this could be the vibration of the cable. However, all errors decrease toward zero during the last 60 seconds of the simulation.

Figure 11 shows the magnitude of cable force acting on each helicopter. The maximum cable force is about $24000 \mathrm{~N}$, which is smaller than the load capacity of K-MAX. The cable force remains constant for most of the time except during the initialization, acceleration, and deceleration period. During the take off period, the upwards component of cable force increases to lift the payload, and then decreases to reduce the vertical velocity to zero. During the North direction acceleration period, the leading helicopters (3 and 4) have to increase the North component of the cable force, and the rear helicopters (1 and 2) have to decrease cable force to maintain the vertical position. This result in the observed non-zero pitch angle. The opposite behavior happens during the deceleration period.

Figure 12 shows the relative distance between each helicopter. The actual helicopter distance is at most 0.5 meter smaller than the desire separation distance. This error is acceptable and within the margin of safety for operation. This result shows that proposed approach satisfied the helicopter separation constraint for safety operation while controlled the payload to follow its desire trajectory. 

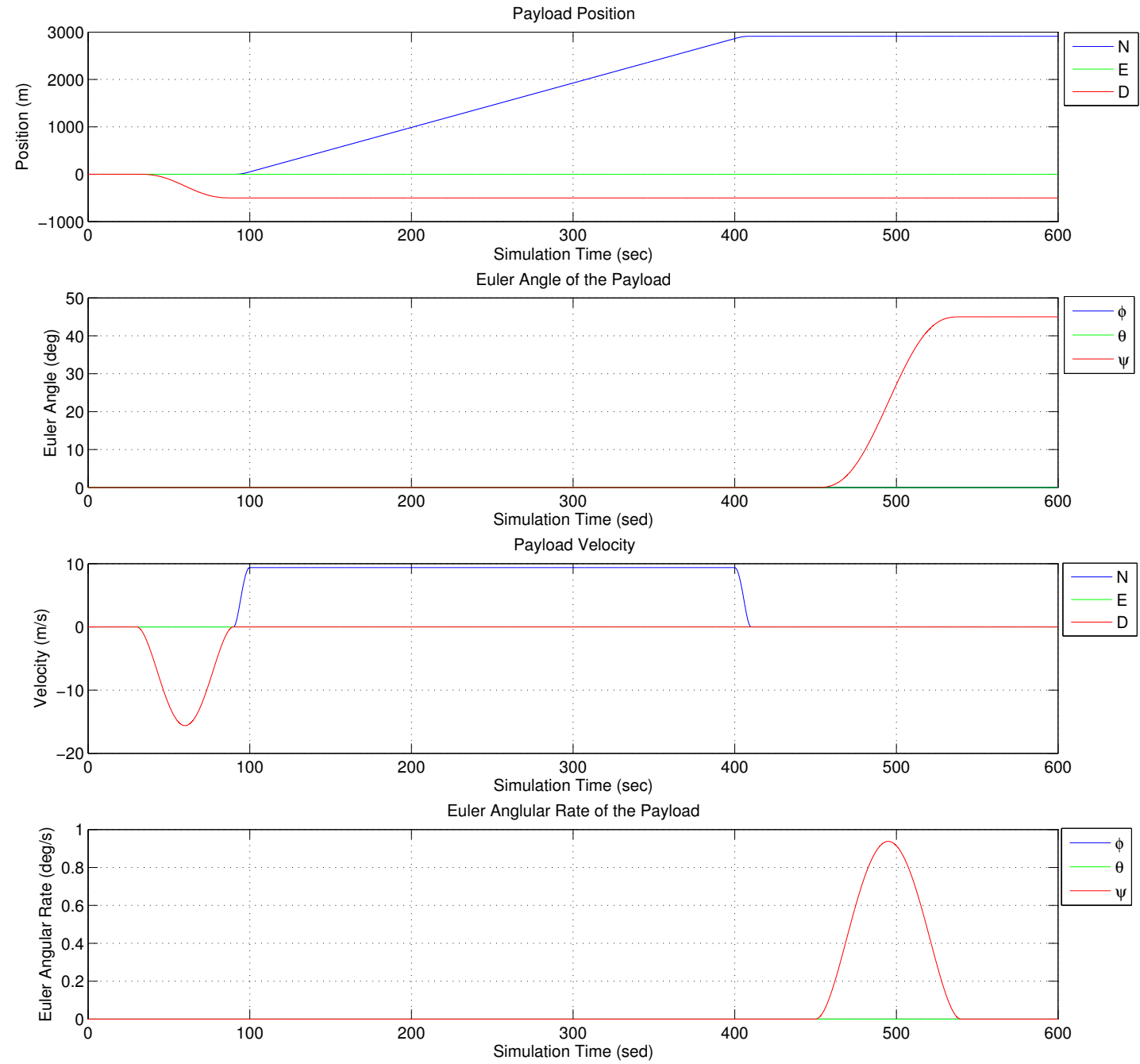

Figure 9. Desired payload state for combined translation/rotation case 

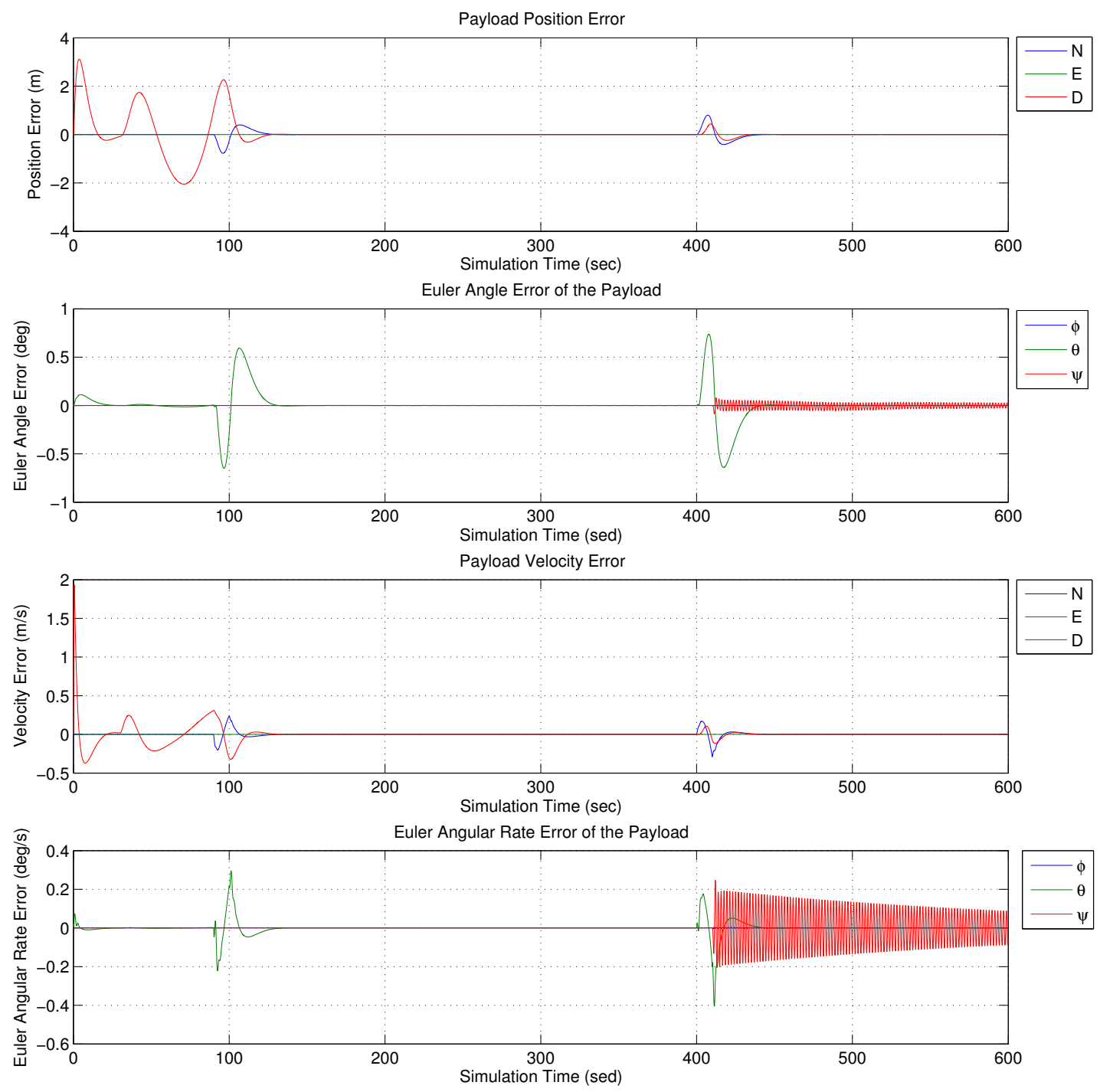

Figure 10. Payload state error for combined translation/rotation case 

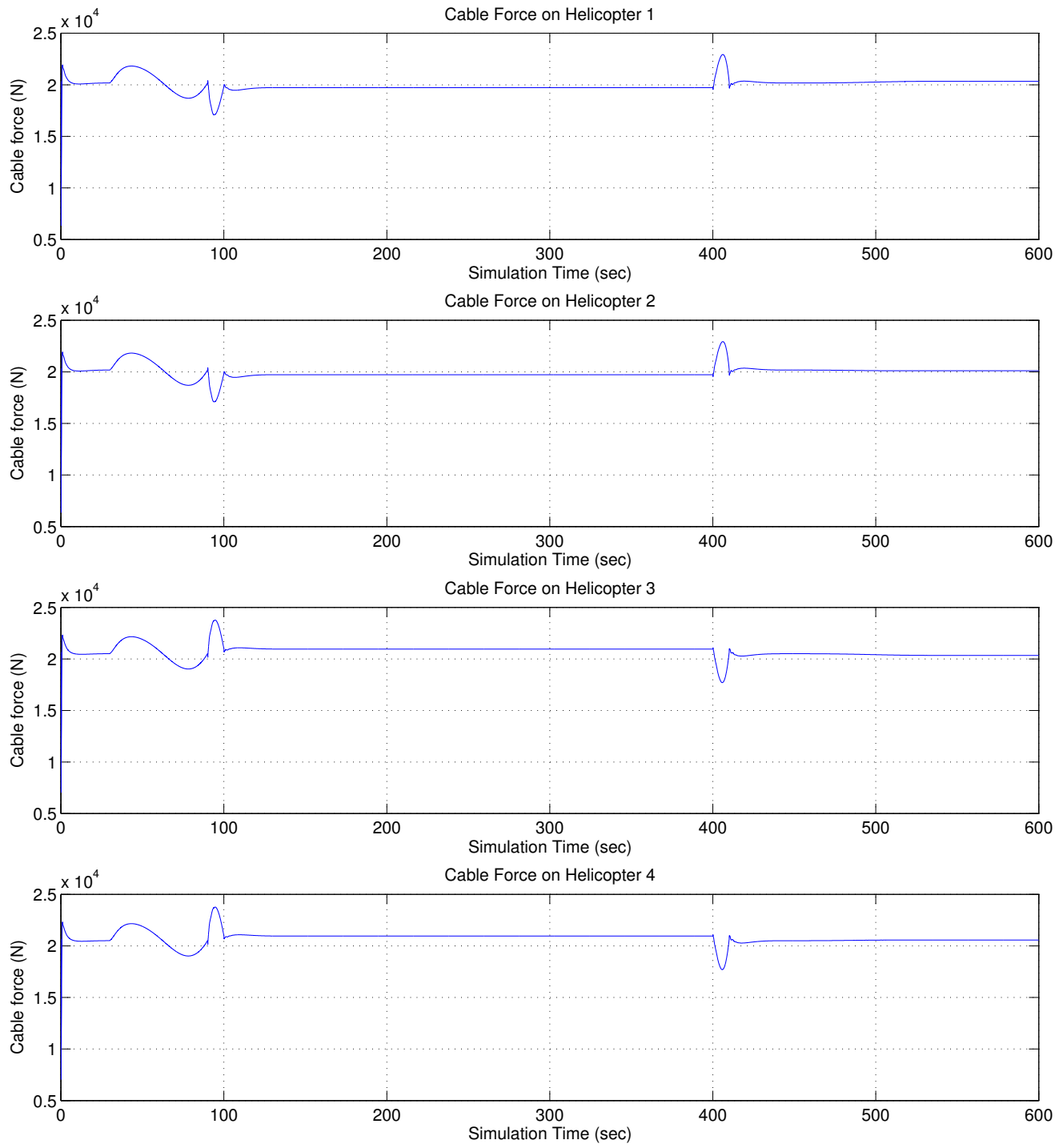

Figure 11. Cable forces for combined translation/rotation case 

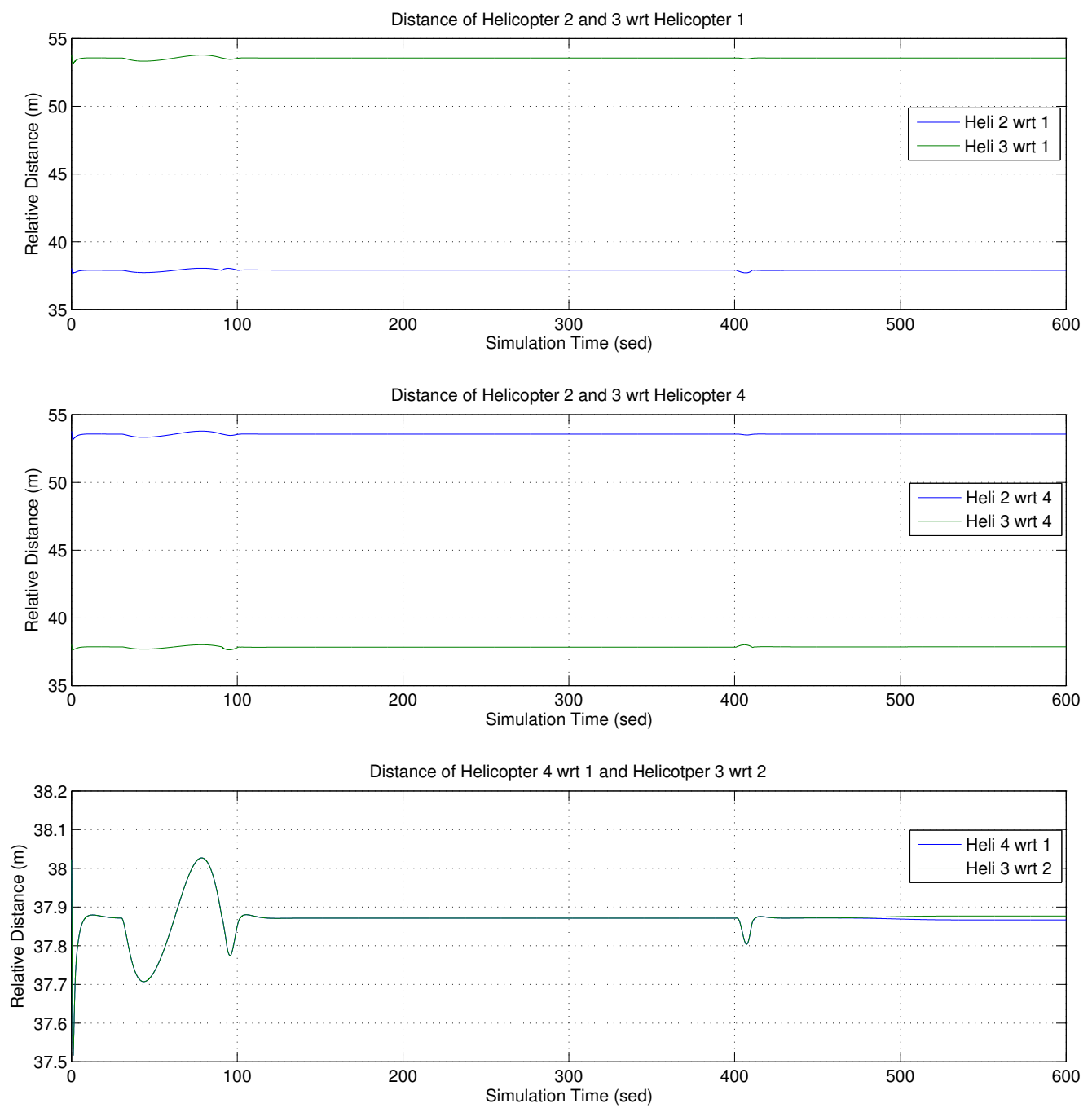

Figure 12. Helicopter relative distance for combined translation/rotation case 


\section{B. Constant Wind Disturbance}

The following cases investigate the effect of a sudden constant wind (a sharp gust) on the payload. Since a lighter payload is more easily disturbed by the wind, payload of mass of $500 \mathrm{~kg}$ was used in these simulations (to increase the effect of disturbances)a. For all following cases, a cable diameter of $6.4 \mathrm{~mm}$ and cable damping constant of $9864.8 \mathrm{~N} * \mathrm{~s} / \mathrm{m}$ were used; the payload was commanded to maintain its desired position at $[0,0,0]$; a constant wind of $33.5 \mathrm{mph}$ in South direction was applied at time 10 seconds.

Two cases were examined to assess the effect of the helicopter separation constraint on performance: 38 meter minimum separation and 40 meter minimum separation.

The results for the 38 meter separation case are shown in Figure 13 to Figure 15.
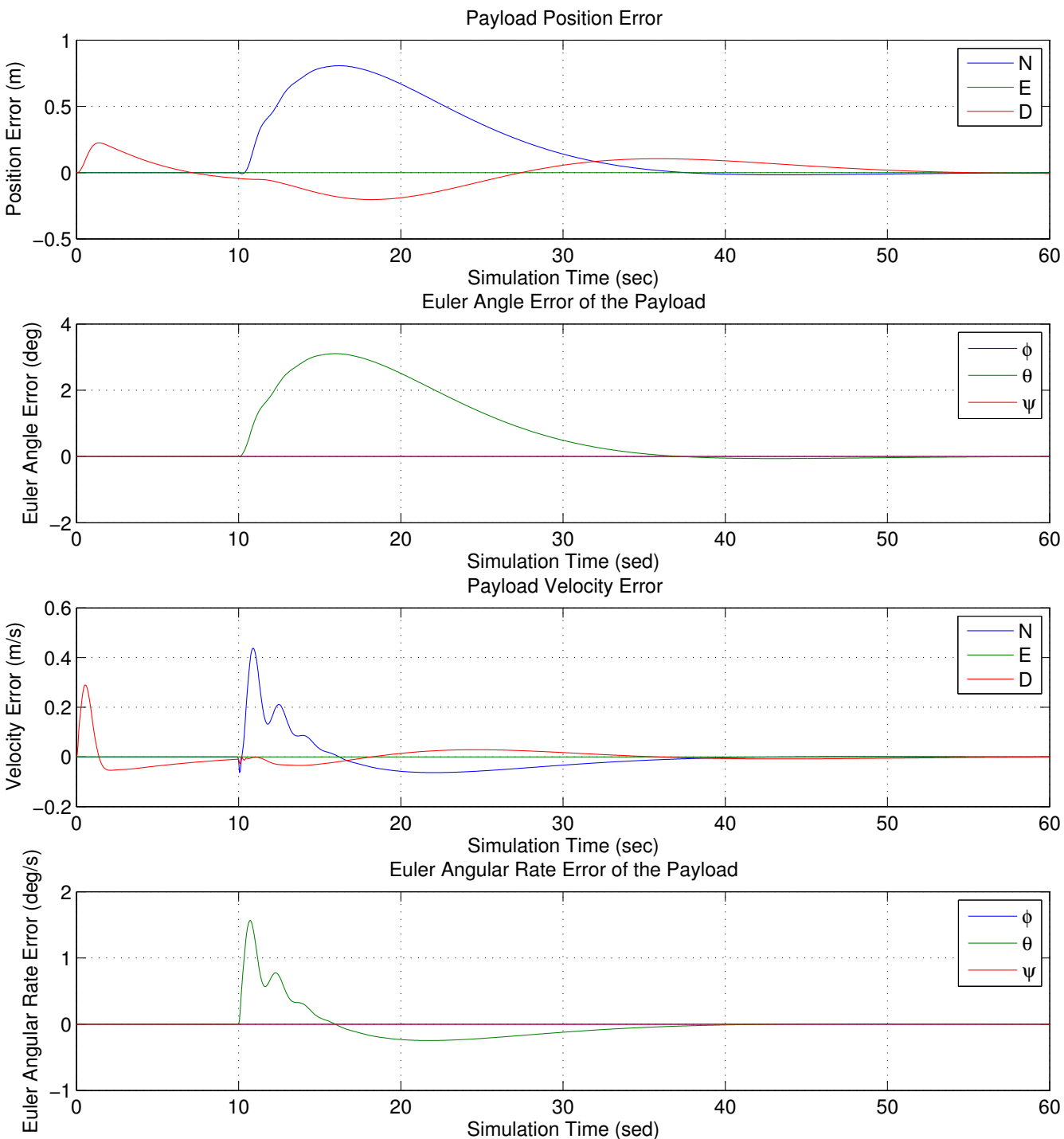

Figure 13. Payload stat errors for helicopter separation of 38 meters

From Figure 13, the payload pitched up and moved Northward as the constant wind suddenly applied. The constant wind applied a constant force on the payload in South direction so the cables have to apply equal amount of net force in North direction to encounter this disturbance. From Figure 14, cable 3 and 4 

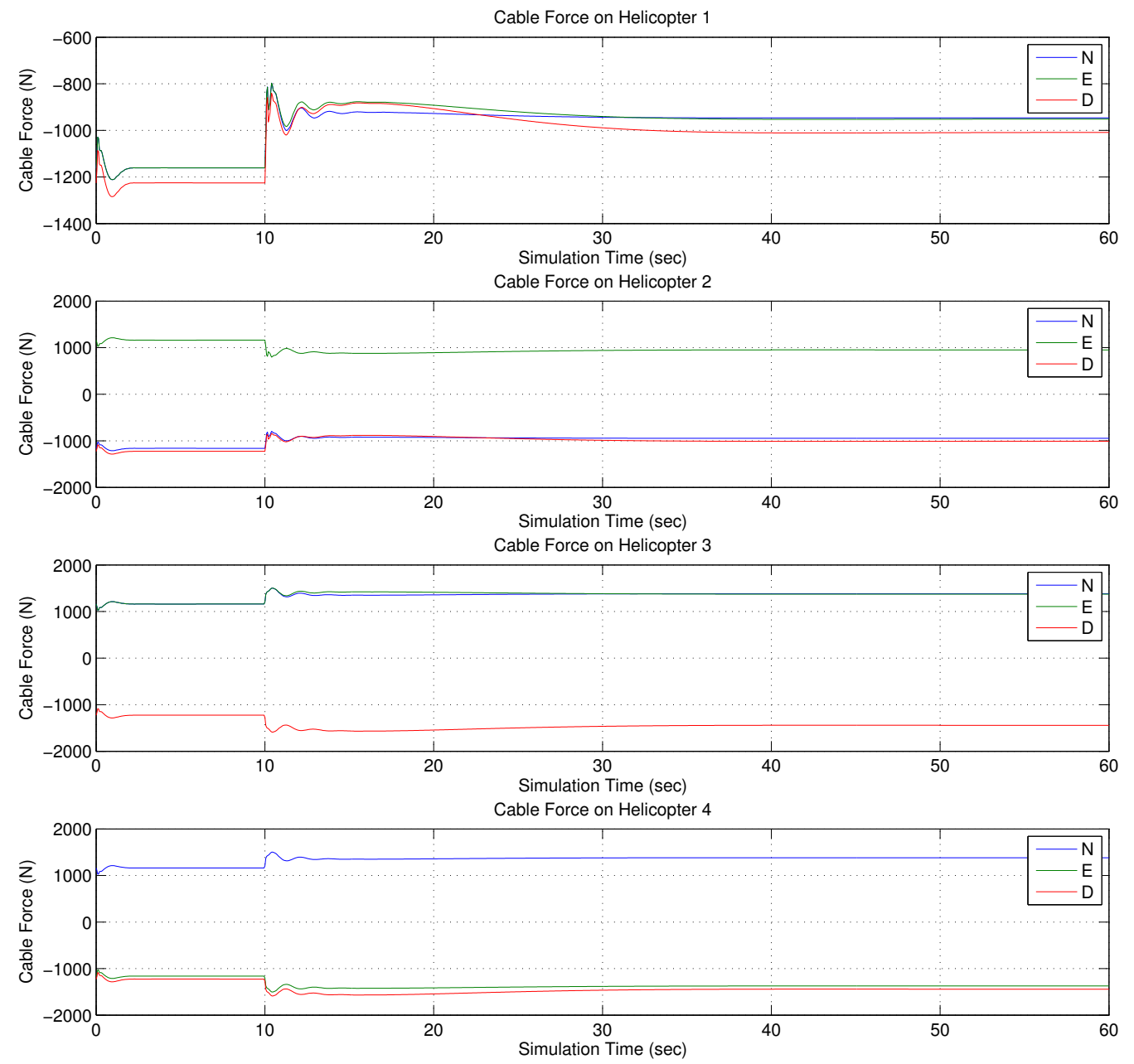

Figure 14. Cable force for helicopter separation of 38 meters

increase all components in magnitude, and cable 1 and 2 decrease all components in magnitude to increase the North component of net force while trying to keep the East and Down component of net force remain unchanged.

As shown in Figure 15, the leading helicopters (3 and 4) flew upward to increase the cable force, and helicopters ( 1 and 2 ) flew downward to decrease the Down component of net force. Therefore the payload pitch angle increased. Pitch error reached a maximum of 3 degrees and then started decrease to zero. Errors in translation dynamic are also cause by the action described above.

The results for the 40 meter separation case are shown in Figure 16 to Figure 18.

From Figure 16, the payload pitched down instead of up, and the error in North position is larger compare to the last case. As shown in Figure 17, cable 3 and 4 increased all components in magnitude at simulation time equal to 10 seconds, but East and Down components started decreasing in magnitude from 10 second to 20 second. Similar but opposite behavior for cable 1 and 2. This shows that helicopter 3 and 4 were flying lower and helicopter 1 and 2 were flying higher, which were also showed in Figure 18. Therefore the payload pitched downward.

The difference in payload behavior for difference helicopter separation constraint distance could be the result of the cost function that was used in cable force computation. In the cases present here, the algorithm 

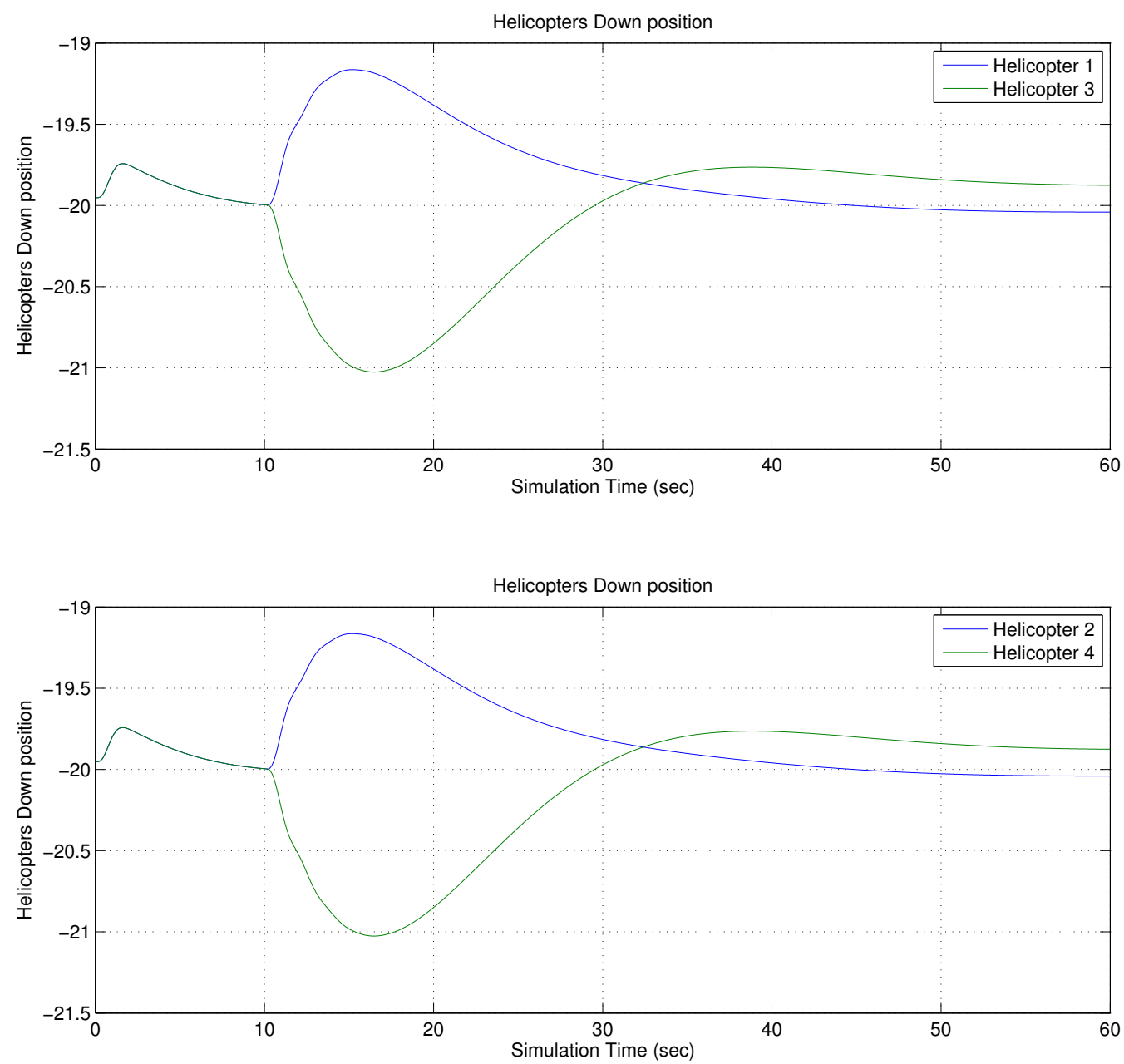

Figure 15. Helicopters Down direction position for 38 meter separation constraint

determined a set of cable force that will minimize the total cable force and satisfy the separation constraint.

Based on the results from last two cases, we assume that there is one value of helicopter separation constraint distance will result in smallest maximum payload pitch error due to the constant wind disturbance. Figure 19 is a plot that shows the relationship between payload maximum pitch angle error and helicopter separation constraint distance with cost function that minimize the total cable force.

The the range of helicopter separation constraint distance for stable operation is from 37.65 to 41.55 . This range may change with different cost function for cable force computation. From Figure 19, the helicopter separation constraint distance that results in smallest maximum payload pitch error is about 38.62 meters. Further studies will be conduct to investigate how is the optimal separation constraint distance relate to payload properties, cable properties, attachment point location, and the cost function used to compute the cable forces.

\section{Conclusion}

This paper has presented a hierarchical approach to coordinated transport of a slung load by a team of autonomous rotorcraft. The approach is based on the concept of object based task level control, which abstracts control into several layers, with a high-level controller computing the desired forces to ensure that 

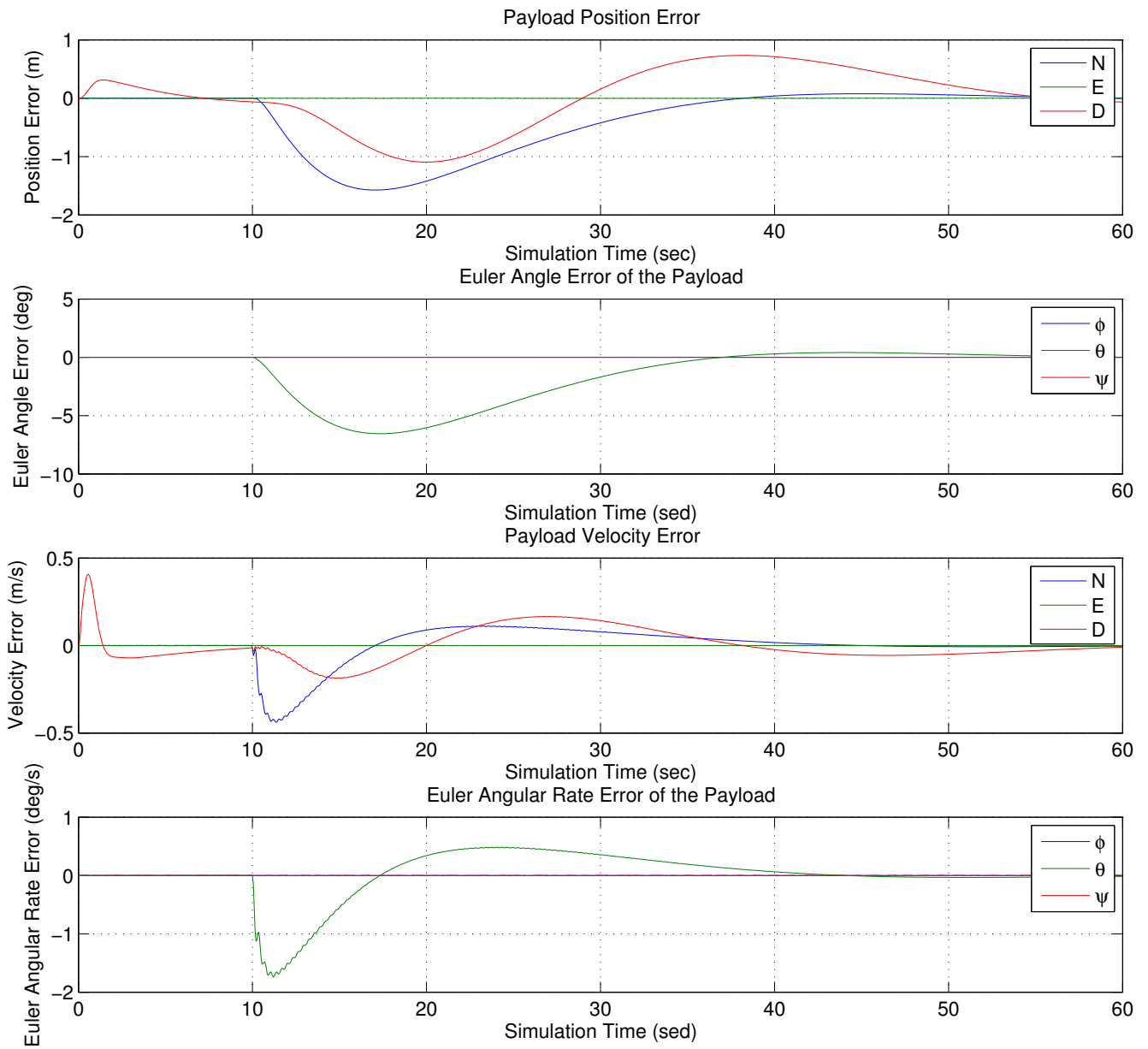

Figure 16. Payload stat errors for helicopter separation of 40 meters

the payload follows a desired trajectory. A lower level controller computes the required cable forces and inner loop controllers on board each helicopter ensure that these desired cable forces are maintained.

With three or more cable attachment points a payload can be controlled in six degrees of freedom, and the resulting null space is used to ensure that other constraints (vehicle separation, payload controllability and disturbance rejection) are satisfied.

This paper has used a linear state feedback controller combined with a feed forward of desired acceleration as a payload trajectory following controller. A two step approach is used to compute cable forces: first, the least norm solution ensures that net forces on the payload are satisfied; second, null space forces are computed so that constraints (here, vehicle separation) are satisfied.

Simulation results of payload transport using four helicopters and cables modeled as damped springs show the utility of the approach. Payload state error remains small and disturbance rejection (response to a step gust) is good.

\section{Acknowledgments}

This research was partially funded by the Government under Agreement No. W911W6-11-2-0011. The U.S Government is authorized to reproduce and distribute reprints notwithstanding any copyright notation thereon. The views and conclusions contained in this document are those of the authors and should not 

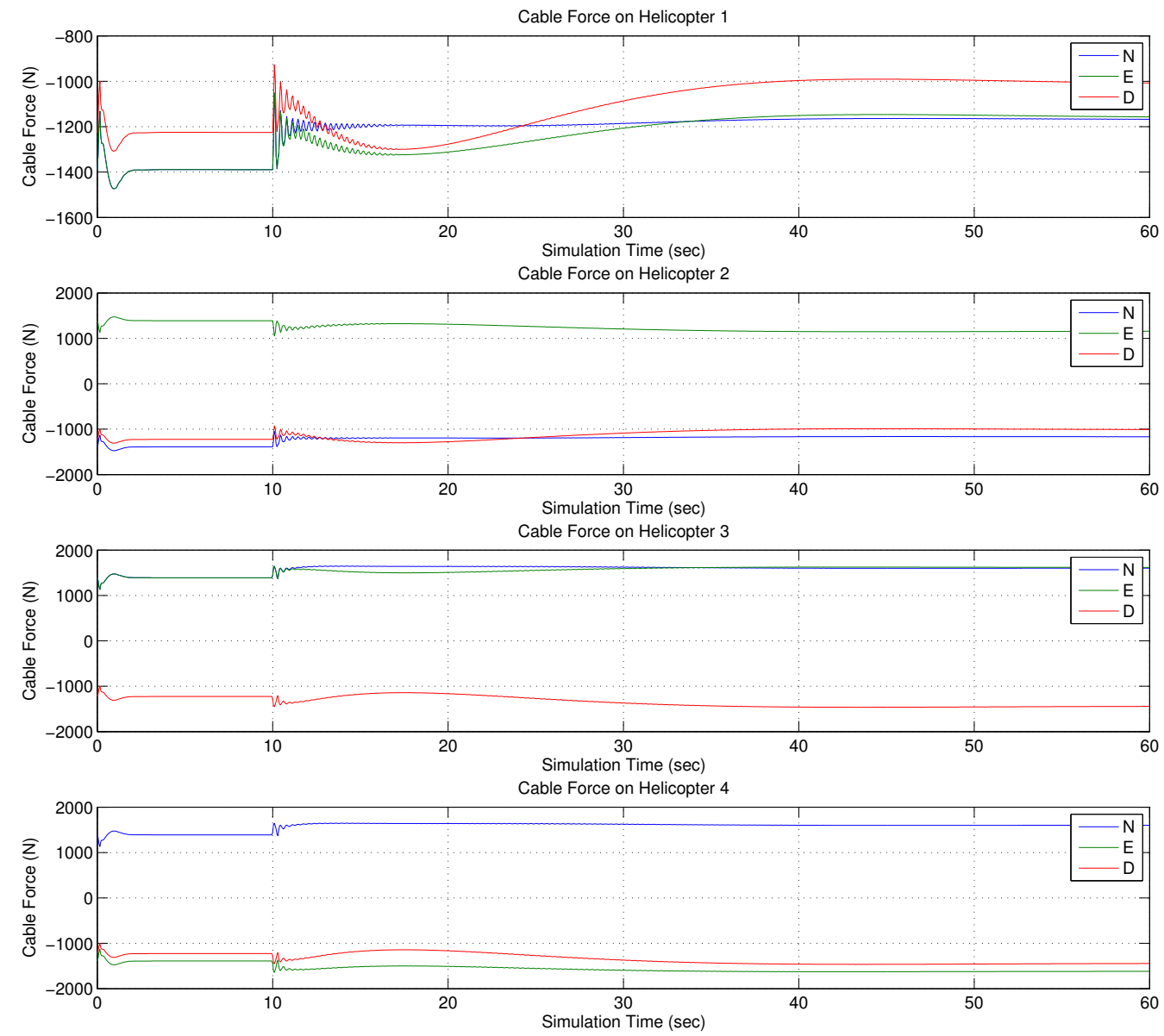

Figure 17. Cable force for helicopter separation of 40 meters

be interpreted as representing the official policies, either expressed or implied, of the U.S. Government.The authors also thank Thanan Yomchinda for providing the helicopter dynamic inversion control law used in the simulations.

\section{References}

${ }^{1}$ Curtiss, H. C. and Warburton, F. W., "Stability and Control of the Twin-Lift Helicopter System," Journal of the American Helicopter Society, Vol. 30, No. 2, April 1985, pp. 14-23.

${ }^{2}$ Mittal, M., Prasad, J. V. R., and Schrage, D. P., "Comparison of Stability and Control Characteristics of Two Twin-Lift Helicopter Configurations," Journal of Nonlinear Dynamics, Vol. 3, No. 3, 1992, pp. 199-223.

${ }^{3}$ Mittal, M. and Prasad, J. V. R., "Three-Dimensional Modeling and Control of a Twin-lift Helicopter System," Journal of Guidance, Control and Dynamics, Vol. 16, No. 1, 1993, pp. 86-95.

${ }^{4}$ Maza, I., Kondak, K., and Bernard, M., "Multi-UAV Cooperation and Control for Load Transportation and Deployment," Journal of Intelligent and Robotic Systems, Vol. 57, No. 1, 2010, pp. 417-449.

${ }^{5}$ Cicolani, L. S. and Kanning, G., "Equations of Motion of Slung-Load Systems, Including Multilift Systems," Tech. Rep. TM-1038798, NASA, September 1992.

${ }^{6}$ Cicolani, L. S. and Kanning, G., "General equilibrium Characteristics of a Helicopter Dual-Lift System," Tech. Rep. TP-2615, NASA, July 1986.

${ }^{7}$ Cicolani, L. S. and Kanning, G., "Equations of Motion of Slung Load Systems with Results for Dual Lift," Tech. Rep. TM-102246, NASA, February 1990. 

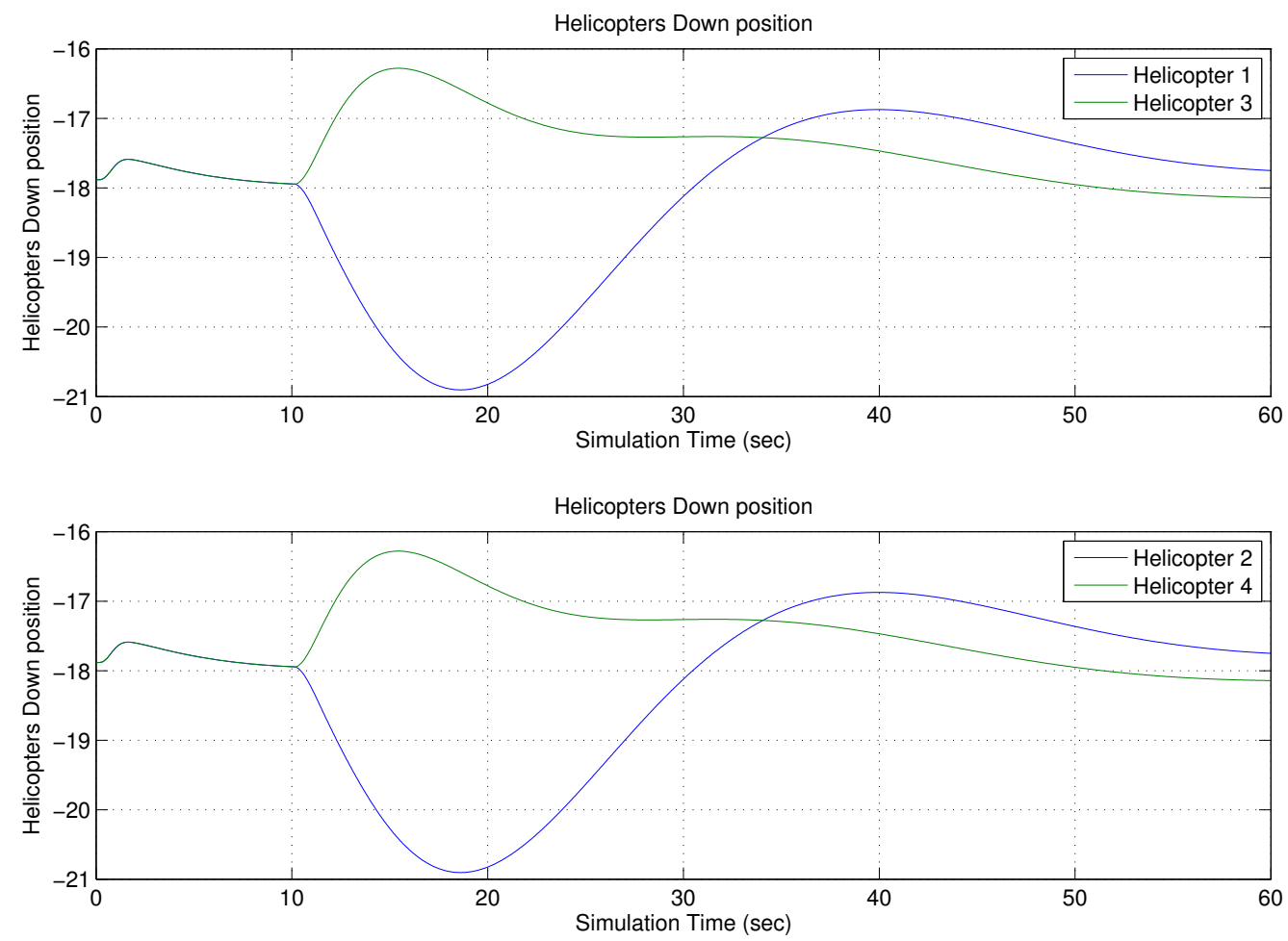

Figure 18. Helicopters Down direction position for 40 meter separation constraint

${ }^{8}$ Rodriquez, A. A. and Athans, M., "Multivariable Control of a Twin Lift Helicopter System using the LQG/LTR Design Methodology," American Controls Conference, June 1986, pp. 1325-1332.

${ }^{9}$ Menon, P. K., Prasad, J. V. R., and Schrage, D. P., "Nonlinear Control of a Twin-Lift Helicopter Configuration," Journal of Guidance, Control and Dynamics, Vol. 14, No. 6, 1991, pp. 1287-1293.

${ }^{10}$ Prasad, J. V. R., Mittal, M., and Schrage, D. P., "Control of a Twin-Lift Helicopter System using Nonlinear State Feedback," Journal of the American Helicopter Society, Vol. 36, No. 4, 1991, pp. 57-65.

${ }^{11}$ Mittal, M., Prasad, J. V. R., and Schrage, D. P., "Nonlinear Adaptve Control of a Twin Lift Helicopter System," IEEE Control Systems, Vol. 11, No. 3, 1991, pp. 39-45.

${ }^{12}$ Stevens, H. D., S., M. E., S.M., R., and Cannon, R., "Object-Based Task-Level Control: A Hierarchical Control Architecture for Remote Operation of Space Robots," Proceedings of the AIAA/NASA Conference on Intelligent Robotics in Field, Factory, Service, and Space, 1994.

${ }^{13}$ Song, Y., Horn, J. F., Li, Z., and Langelaan, J. W., "Modeling, Simulation and Non-linear Control of a Rotorcraft Multi-lift System," American Helicopter Society 69th Annual Forum, American Helicopter Society, Phoenix, AZ, May 21-23 2013.

14 "K-MAX Performance and Specs," http://www.kaman.com, Accessed: 20/11/2013. 
Payload Pitch error vs. helicopter separation constraint distance

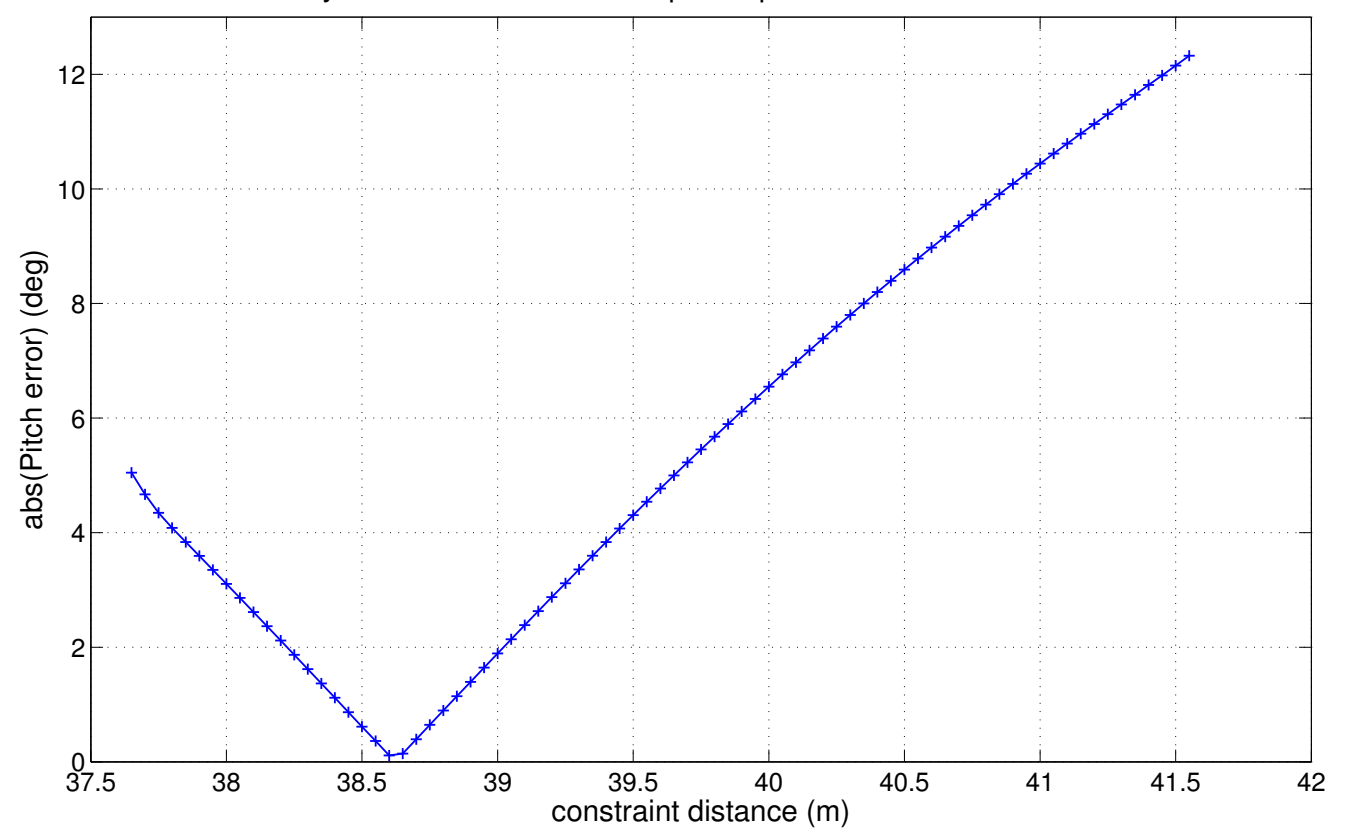

Figure 19. Payload pitch error vs. helicopter separation constraint 